\title{
Laser-Induced Isotopic Effects in Titanium Resonance Ionization
}

\author{
R. K. Wunderlich, ${ }^{\dagger}$ G. J. Wasserburg, ${ }^{\ddagger}$ I. D. Hutcheon, ${ }^{, \ddagger}$ and Geoffrey A. Blake \\ Division of Geological and Planetary Sciences, California Institute of Technology, Mail Code 170-25, \\ Pasadena, California 91125
}

\begin{abstract}
Titanium isotope ratios have been measured by resonance ionization mass spectrometry (RIMS) with special emphasis on the nature of laserinduced isotopic selectivity. A pronounced wavelength dependence of even mass isotope ratios is caused by large nuclear volume effects near the magic neutron number 28 in ${ }^{30} \mathrm{Ti}$. Optical isotope shifts, ranging from 0.07 to $0.21 \mathrm{~cm}^{-1}$, between ${ }^{50} \mathrm{Ti}$ and ${ }^{46} \mathrm{Ti}$ were measured for several transitions. The ${ }^{50} \mathrm{Ti} /{ }^{46} \mathrm{Ti}$ and ${ }^{48} \mathrm{Ti} /{ }^{46} \mathrm{Ti}$ ratios, nevertheless, exhibited only mass-dependent fractionation, in which the lighter $T i$ isotopes were enriched by $\sim 2.5 \%$ /amu, when the laser operating parameters were properly controlled. Odd-even mass isotopic selectivity in the resonant ionization process was also examined for several transitions as a function of the laser polarization state and intensity. Under saturating conditions for a $\Delta J=+1$ transition and a high degree of laser depolarization for a $\Delta J=0$ transition, the odd-even isotopic enhancement was reduced below the $2 \%$ level. The Ti isotope data agree with our previous results for $O s$ and indicate that, by a careful choice of resonance transitions and laser operating parameters, isotope ratios can be measured accurately and reliably with RIMS.
\end{abstract}

\section{INTRODUCTION}

The high elemental selectivity ${ }^{1}$ and sensitivity ${ }^{2-4}$ of resonance ionization mass spectrometry (RIMS) gives this technique the potential to overcome many of the limitations of secondary ion mass spectrometry and to provide a substantial advance in elemental and isotopic in situ microanalysis. The application of RIMS to high precision isotopic analysis is, however, complicated by the presence of laser-induced isotopic selectivity in the resonant photoionization process. While in some cases little or no laser-induced isotopic selectivity is observed, ${ }^{5}$ in most studies the recorded shifts in measured isotopic ratios, compared to the true sample composition,

\footnotetext{
+Present address: Physikalisches Institut, Universität Augsburg, Augsburg, Germany.

Also with the Charles Arms Laboratory, California Institute of Technology.

Also with the Beckman Institute, California Institute of Technology.

(1) Hurst, G. S.; Payne, M. G.; Nayfeh, N. H.; Judish, J. P.; Wagner, E. B. Phys, Rev. Lett. 1975, 35, 82-85.

(2) Parks, J. E.; Spaar, M. T.; Beekman, D. W.; Moore, L. J.; Cressman, P. J. Resonance Ionization Spectroscopy 1988. Inst. Phys. Conf. Ser. 1988, No. 94, 197-200.

(3) Pellin, M. J.; Young,C. E.; Calaway, W.F.; Burnett, J.W.; Jorgensen, B.; Schweitzer, E. L.; Gruen, D. M. Nucl. Instrum. Methods Phys. Res. $1987, B 18,446-451$.

(4) Blum, J. D.; Pellin, M. J.; Calaway, W. F.; Young, C. E.; Gruen, D. M.; Hutcheon, I. D.; Wasserburg, G. J. Anal. Chem. 1990, 62, 209-214. (5) Fassett, J. D.; Powell, L. J.; Moore, L. J. Anal. Chem. 1984, 58, $2923-2926$.
}

range from $2-3 \%^{6}$ to nearly $100 \%,{ }^{7-13}$ typically with an enhancement of the odd mass isotopes. For example, in a recent RIMS study of $\mathrm{Ti}$, laser-induced enrichments in the ${ }^{47} \mathrm{Ti} /{ }^{48} \mathrm{Ti}$ and ${ }^{49} \mathrm{Ti} /{ }^{48} \mathrm{Ti}$ ratios of up to $35 \%$ and $48 \%$, respectively, were observed. ${ }^{10,11}$ In many cases these isotopic shifts cannot be explained solely by laser bandwidth effects (that is, effects induced when the laser bandwidth is less than the width of the hyperfine transition array of the odd mass isotopes) or by selection rule differences in ionization efficiency between odd and even mass isotopes. Large shifts in measured isotope ratios also hinder the recognition of true isotopic abundance variations and require frequent analyses of standards to monitor and remove laser-induced isotopic bias. ${ }^{11}$

The accuracy and reliability of isotope ratios obtained by RIMS may thus depend critically on the availability of suitable standards as well as on the relative sizes of isotopic effects indigenous to the sample and laser-induced isotopic selectivity. Accurate Ti isotope ratios, for example, have been determined using RIMS in the presence of large even-odd effects by expressing the (unknown) $\mathrm{Ti}$ isotope ratios in the sample relative to the same ratio measured in a standard in the same experiment. ${ }^{10,11}$ However, in many important isotope systems in geochemistry, particularly those involving radiogenic isotopes, this ratioing technique may not be so successful. Indeed, in the $\mathrm{Re}-\mathrm{Os}$ system the ability to remove even-odd isotope effects by reference to a standard is limited by the magnitude of the (poorly constrained) radiogenic contribution to the ${ }^{187} \mathrm{Os}$ abundance. In this case laserinduced odd-even effects must be inferred from the measured abundance of the nonradiogenic ${ }^{189} \mathrm{Os}$ isotope. Because of the dynamic nature of the resonance ionization process discussed below and the different nuclear spins of ${ }^{187} \mathrm{Os}$ and ${ }^{189} \mathrm{Os}$, correction factors obtained for ${ }^{189} \mathrm{Os}$ may not be directly applicable to ${ }^{187} \mathrm{Os}^{6}$

Three sources of laser-induced isotopic effects can be distinguished. (i) Laser bandwidth and tuning effects: In the presence of optical isotope shifts and hyperfine structure, isotope biases measured with RIMS will depend on the precise

(6) Walker, R. J.; Fassett, J. D. Anal. Chem. 1986, 58, 2923-2927.

(7) Young, J. P.; Shaw, R. W.; Goeringer, D. E.; Smith, D. H. Resonance Ionization Spectroscopy 1988. Inst. Phys. Conf. Ser. 1988, No. 94, 367370.

(8) Miller, C. M.; Fearey, B. L.; Palmer, B. A.; Nogar, N. S. Resonance Ionization Spectroscopy 1988. Inst. Phys. Conf. Ser. 1988, No. 94, 297306.

(9) Fairbank, W. M., Jr.; Spaar, M. T.; Parks, J. E.; Hutchinson, J. M. Phys. Rev. A 1989, 40, 2195-2198.

(10) Spiegel, D. R.; Pellin, M. J.; Calaway, W. F.; Burnett, J. W.; Coon, S. R.; Young, C. E.; Gruen, D. M.; Clayton, R. N. Lunar Planet. Sci. 1991, 22, 1303-1304.

(11) Spiegel, D. R.; Calaway, W. F.; Davis, A. M.; Burnett, J. W.; Pellin, M. J.; Coon, S. R.; Young, C. E.; Clayton, R. N.; Gruen, D. M. Anal. Chem. $1992,64,469-475$.

(12) Feary, B. L.; Tissue, B. M.; Oliveras, J. A.; Loge, G. W.; Murrell M. T.; Miller, C. M. Presented at the VI International Symposium on Resonance Ionization Spectroscopy and Its Applications, Sante $\mathrm{Fe}, \mathrm{NM}$, May 24-29, 1992.

(13) Hayman, C.; Comaskey, B.; Johnson, M.; Worden, W. SPIE Proc. $1988,912,200-204$ 
laser wavelength and resetability, ${ }^{12}$ bandwidth, and intensity. The evaluation of the wavelength dependence of isotope ratios is a prerequisite for the measurement of reproducible and accurate isotope ratios by RIMS and for the unambiguous identification of other sources of laser-induced isotope effects. (ii) Dynamic effects: Dynamic effects arise from the detailed time evolution of the interaction between the laser radiation and the atomic system, ${ }^{14-17}$ including quantum mechanical coherence, and lead to a dependence of measured isotope ratios on the degree of saturation of the ionization and on the laser pulse duration. (iii) Selection rule and polarization effects: Selection rule and polarization effects can produce large variations in sensitivity of the excitation-ionization process for even vs odd mass isotopes, causing isotope ratios to depend on the nature of the discrete transition and on the polarization state of the laser radiation. ${ }^{18,19}$

The processes leading to laser-induced isotopic effects have been addressed in several recent theoretical ${ }^{14-17}$ and experimental studies. ${ }^{18}$ Wunderlich et al., ${ }^{18}$ for example, demonstrated several effects in the resonance ionization of $O s$ which can cause isotope selectivity and devised methods to reduce them below the $1 \%$ level. Here, we apply some of these methods to the case of $\mathrm{Ti}$ in order to determine whether or not they can be generally applied to significantly reduce laserinduced isotopic selectivity in RIMS. We have chosen $\mathrm{Ti}$ because of the important role $\mathrm{Ti}$ isotope measurements have assumed in cosmochemistry as an indicator of nucleosynthetic components from different stellar sources. ${ }^{20-22}$ In addition, the large even-odd isotope shifts in a previous RIMS study of $\mathrm{Ti}^{10,11}$ serve as a benchmark against which the effectiveness of our laser tuning procedures may be evaluated.

In the following discussion, we present new data on the resonance ionization of $\mathrm{Ti}$ obtained with a thermal atomization-quadrupole mass spectrometer. These data are used to address the possible sources of laser-induced isotopic selectivity outlined above. We then demonstrate for selected transitions that the application of a wavelength tuning procedure developed for Os allows measurement of eveneven isotope ratios in $\mathrm{Ti}$ which differ from the ratio present in the sample only by purely mass-dependent fractionation, that is, by fractionation which depends only on instrumental factors other than the photoionization process.

\section{EXPERIMENTAL SECTION}

The mass spectrometer, laser system, and experimental procedures for measuring isotope ratios used in this study were similar to those used in our recent work on Os-RIMS and are described in detail by Wunderlich et al. ${ }^{18}$ The mass spectrometer was a Finnigan-MAT thermal ion source quadrupole mass spectrometer modified to accommodate a laser beam in the ion formation region. The mass resolution utilized in these experiments was typically 160 (10\% valley), providing a clear separation of the Ti mass peaks. Ti samples were prepared by melting a small amount of Ti metal powder on a Ta filament ribbon, providing a $T i$ spot approximately $1 \mathrm{~mm}$ in length at the center of the filament. Neutral Ti atoms were emitted from the filament at a temperature of $\sim 1000^{\circ} \mathrm{C}$, intersected by a laser beam passing

(14) Lambopoulos, P.; Lyras, A. A. Phys. Rev. A 1989, 40, 2199-2202

(15) Lyras, A. A.; Zorman, B.; Lambropoulos, P. Phys. Rev. A 1990, 42, $543-549$.

(16) Whitten, W. B.; Ramsey, J. M. Appl. Spectrosc. 1990, 44, 11881192 .

(17) Payne, M. G.; Allman, S. L.; Parks, J. E. Spectrochem. Acta B $1991,46,1439-1457$

(18) Wunderlich, R. K.; Hutcheon, I. D.; Wasserburg, G. J.; Blake, G. A. Int. J. Mass Spectrom. Ion Processes 1992, 115, 123-155.

(19) Balling, L. C.; Wright, Z. Z. Appl. Phys. Lett. 1976, 29, 411-413.

(20) Papanastassiou, D. A. Astrophys. J. 1986, 308, L27-L30.

(21) Papanastassiou, D. A.; Brigham, C. A. Astrophys. J. 1989, 338, L37-L40.

(22) Ireland, T. R.; Zinner, E. K.; Amari, S. Astrophys. J. 1991, 376 , L53-L56.
1-2 $\mathrm{mm}$ in front of the filament, and ionized (see Figure 1 in ref 18 for a detailed view of the ion formation region). At this filament temperature, the production of Ti thermal ions was insignificant, the thermal ion background being less than $0.1 \%$ of the $\mathrm{Ti}^{+}$ resonance ionization signal at any given $\mathrm{Ti}$ mass. Ion currents were measured with an $80-\mathrm{MHz}$ counting system, and isotope ratios were collected in the peak jumping mode of the quadrupole mass spectrometer. The linearity of the counting system was checked by RIMS measurements of the ${ }^{48} \mathrm{Ti} /{ }^{46} \mathrm{Ti}$ isotope ratio as a function of the intensity at mass 48 . No dead-time effects were observed for ${ }^{48} \mathrm{Ti}^{+}$intensities corresponding to a maximum of $\sim 40$ photoions per laser pulse, consistent with our earlier Os results. ${ }^{18}$

The laser system consisted of a Lambda Physik LPX 110iC $\mathrm{XeCl}$ excimer laser, which pumped a Lambda Physik FL3002E tuneable dye laser equipped with a BBO crystal for frequency doubling. The laser system was capable of operating at repetition rates of $200 \mathrm{~Hz}$, but most experiments were performed at $20 \mathrm{~Hz}$ due to increased laser instabilities at higher repetition rates. The reproducibility of the optical beam alignment in the ion extraction region of the quadrupole mass spectrometer was assured by two fixed apertures, which defined the laser beam position relative to the evaporation filament. In this work we examined simple $1+1$ (one photon resonant, two-photon ionization) schemes in the wavelength region between 250 and $270 \mathrm{~nm}$, which is the frequency doubled tuning range of Coumarin 307 and Coumarin 153 laser dyes. Ti has an ionization potential of $6.83 \mathrm{eV}$, so that the resonant states in this region could be ionized both by the dye laser second harmonic and by the fundamental, which had a nominal bandwidth of $0.2 \mathrm{~cm}^{-1}$. The dye laser could also be operated with an intracavity etalon to reduce the bandwidth to $0.04 \mathrm{~cm}^{-1}$ or, alternatively, without an intracavity etalon and in a lower grating order to increase the visible bandwidth to 0.35 $\mathrm{cm}^{-1}$. Laser bandwidths were monitored with an etalon having a free spectral range of $1.7 \mathrm{~cm}^{-1}$ and a finesse of 18 at $500 \mathrm{~nm}$. Without the intracavity etalon no single mode structure could be observed. The Doppler width of the transitions considered here was $<0.10 \mathrm{~cm}^{-1}$. The ability to operate the laser at increased bandwidth was important for the control of isotope ratios in the presence of hyperfine structure and optical isotope shifts, as will be shown below.

The visible and UV laser beams were focused parallel to the filament with a $50-\mathrm{cm}$ focal length lens to a diameter of $0.9-1$ $\mathrm{mm}$. Typical pulse energies measured after transmission through the mass spectrometer were $8 \mathrm{~mJ}$ in the visible and $0.4 \mathrm{~mJ}$ in the UV. With a pulse duration of $20 \mathrm{~ns}$, intensities of $5.1 \times 10^{7}$ $\mathrm{W} / \mathrm{cm}^{2}$ in the visible and $4.5 \times 10^{6} \mathrm{~W} / \mathrm{cm}^{2}$ in the UV for the ionization and resonance excitation laser fields were obtained. These intensities saturate all bound-free transitions possessing photoionization cross sections $>2.5 \times 10^{-18} \mathrm{~cm}^{2}$ and discrete transitions with $g f$ values $>2 \times 10^{-4}$, where $f$ is the transition oscillator strength and $g$ is the degeneracy factor of the lower state.

The presence of isobaric interferences at the $\mathrm{Ti}$ masses, due to nonresonant ionization of impurities evaporating from the filament, was checked by scanning the mass spectrometer in the $38-52$ amu range with the laser tuned to the $\mathrm{Ti} \mid 3 \mathrm{~d}^{2} 4 \mathrm{~s}^{2} \mathrm{a}^{3} \mathrm{~F}_{2}>\rightarrow$ $\left|3 \mathrm{~d}^{3} 4 \mathrm{p} \mathrm{s}^{3} \mathrm{D}_{3}^{\circ}\right\rangle$ resonance at $39715.51 \mathrm{~cm}^{-1}$. No ion signal above background was detected at masses other than those corresponding to Ti at a filament temperature of $1100^{\circ} \mathrm{C}$. In particular, $K$ is expected to be an abundant neutral species at this temperature. The absence of any ion signal above noise, other than $\mathrm{Ti}^{+}$, indicates that the nonresonant ionization of other species was unimportant and that the background was dominated by the small contribution of thermal ions. Simple $1+1$ ionization schemes on $\mathrm{Ti}$ metal were chosen here to enable unambiguous measurements and control of laser-induced isotopic biases. RIMS applied to elemental and isotopic analyses of natural samples may well require more sophisticated ionization schemes to achieve sufficient selectivity.

Figure 1 shows two consecutive scans of the $\left|3 d^{2} 4 s^{2} a^{3} F_{2}\right\rangle \rightarrow$ $\left|3 \mathrm{~d}^{3} 4 \mathrm{p} \mathrm{s}^{3} \mathrm{D}_{3}^{\circ}\right\rangle$ resonance at $39715.51 \mathrm{~cm}^{-1}$ with the mass spectrometer tuned to 46 and 47 amu, respectively. The scans were performed under nonsaturating ionization and excitation laser power conditions and with a visible laser bandwidth of $0.2 \mathrm{~cm}^{-1}$. 

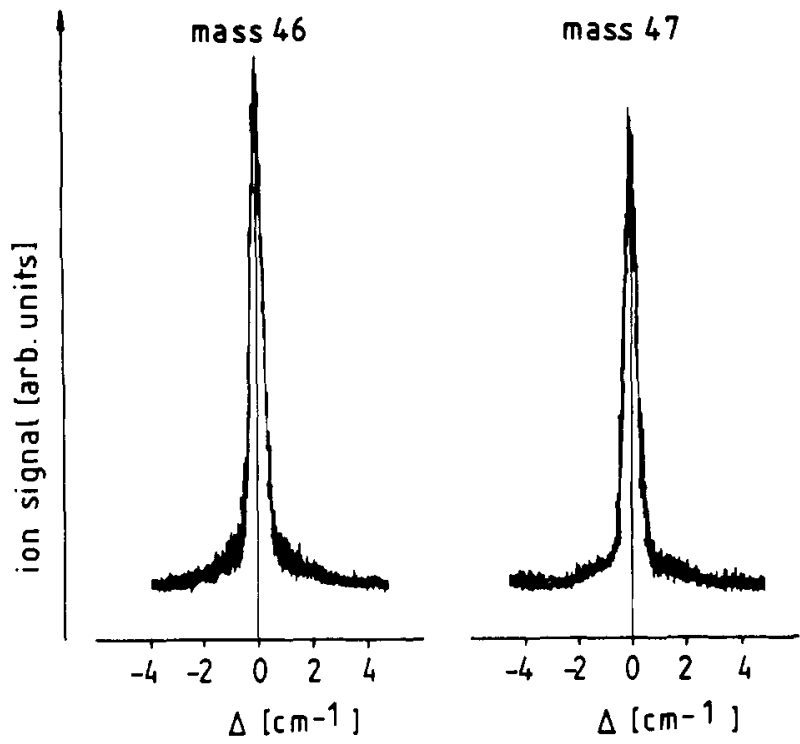

Figure 1. Photolonization line shape of the $\left|3 d^{2} 4 s^{2} a^{3} F_{2}\right\rangle \rightarrow \mid 3 d^{3} 4 p$ $s^{3} D_{3}^{\circ}>$ resonance transition at $39715.51 \mathrm{~cm}^{-1}$ obtained with the mass spectrometer tuned to masses 46 and $47 \mathrm{amu}$. The visible laser bandwldth was $0.2 \mathrm{~cm}^{-1}$.

No hyperfine structure is revealed in the spectrum at mass 47 . Similar spectra were recorded on each transition studied in order to ensure that all odd mass isotope HFS components could be excited simultaneously. The line shapes are structureless and symmetric, further indicating the absence of nonresonant ionization. Because $\mathrm{Ti}$ has a rather high density of states in the 250-270-nm spectral region, the dye laser wavelength was calibrated with $\mathrm{Re}$ resonance ionization signals from a blank $\mathrm{Re}$ filament, which provides several well-separated transitions. The calibration accuracy was $0.3 \mathrm{~cm}^{-1}$.

The saturation behavior of the discrete transitions was checked by observing the ionization signal as a function of the detuning of the frequency doubling crystal from the optimum phasematching tilt angle. All transitions studied could be saturated with the UV intensities available in this experiment. The saturation of the ionization was measured by increasing the pump laser intensity while tuning the frequency doubling crystal for constant UV output at approximately $5 \times 10^{5} \mathrm{~W} / \mathrm{cm}^{2}$.

\section{WAVELENGTH DEPENDENCE, OPTICAL ISOTOPE SHIFTS, AND MASS FRACTIONATION}

Whenever the efficiency (the product of fractional ionization and instrument transmission) of mass spectrometric analysis is less than unity, the measured ratio of ion currents for two isotopes $i$ and $j, R_{\mathrm{M}}^{i j}$, will differ from the true abundance ratio, $R_{0}^{i j}$, due to mass-dependent fractionation arising during ionization and detection. In mass spectrometric analysis with conventional ion sources $R_{M}^{i j}$ may be related to a standard reference value, $R_{\mathrm{S}}^{i j}$, by a fractionation law of the form

$$
R_{\mathrm{M}}^{i j}=f(\alpha) R_{\mathrm{S}}^{i j}
$$

The exact nature of $f(\alpha)$ is, in general, not known but typically is approximated by either a linear law, $1+m_{i j} \alpha$, a power law, $(1+\alpha)^{m_{i j}}$, or an exponential law, $\left(m_{i} / m_{j}\right)^{\alpha}$, where $m_{i j}=m_{i}-$ $m_{j}$ and $\alpha$ is the fractionation per unit mass. ${ }^{23,24}$ For purely mass-dependent fractionation, $\alpha$ is mass independent and common practice involves choosing a standard ratio $R_{S}^{i j}$ and calculating the fractionation factor $\alpha$ from a suitable measured

(23) Wasserburg, G. J.; Jacobson, J. B.; DeProlo, D. J.; McColloch, M. T.; Wen, T. Geochim. Cosmochim. Acta 1981, 45, 2311-2323.

(24) Hart, S. R.; Zindler, A. Int. J. Mass Spectrom. Ion Processes $1989,89,287-301$.

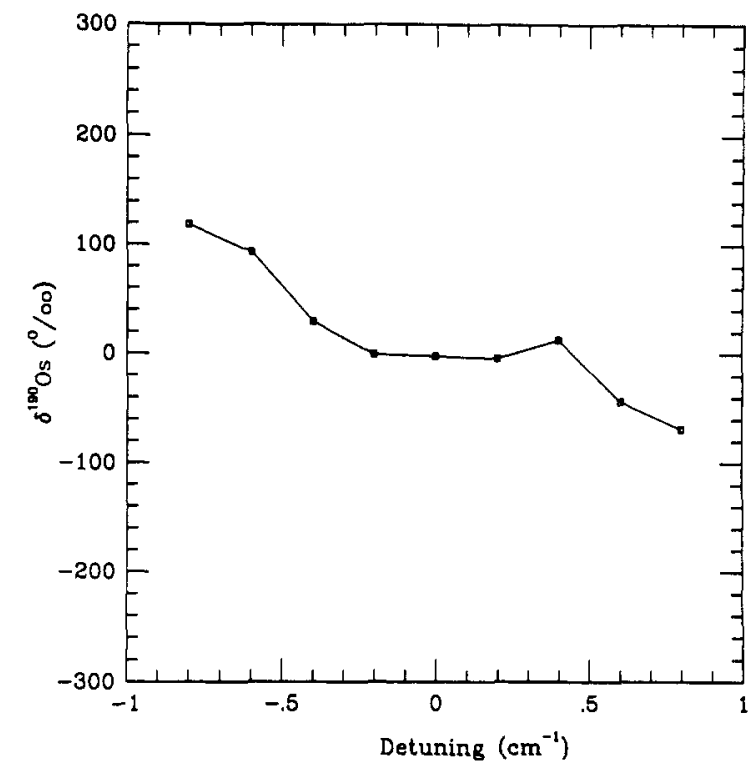

Figure 2. Wavelength dependence of the ${ }^{190} \mathrm{Os} /{ }^{188} \mathrm{Os}$ lsotope ratio measured for the $39406.9 \mathrm{~cm}^{-1}, J=5$ resonance state; $\delta^{190} \mathrm{Os}$ (\%) $=\left[\left({ }^{190} \mathrm{Os} /{ }^{188} \mathrm{Os}\right)_{\text {moas }} /\left({ }^{190} \mathrm{Os} /{ }^{188} \mathrm{Os}\right)_{\mathrm{sTD}}-1\right] \times 1000,\left({ }^{190} \mathrm{Os} /{ }^{188} \mathrm{Os}\right)_{\mathrm{sTD}}=$ $1.9905 ; 25$ oscillator strength $f<0.003 ; \Delta \omega_{\mathrm{L}}=0.70 \mathrm{~cm}^{-1}$. Typlcal error of the individual data points is $1 \%<1 \sigma<1.5 \%$.

ratio $R_{\mathrm{M}^{\text {. }}}^{i j}$ This value of $\alpha$ is then used to calculate ratios $R^{k j}$, corrected for instrumental fractionation. The application of this procedure to isotope ratio measurements in RIMS is complicated by the presence of optical isotope shifts (IS) and hyperfine structure (HFS), both of which may cause measured isotopic ratios to depend on the precise laser wavelength setting, bandwidth, and intensity, ${ }^{18}$ introducing large uncertainties into the mass fractionation correction. It is, therefore, essential to obtain conditions with regard to the laser bandwidth, intensity, and wavelength reproducibility for which a mass-independent fractionation law may be used to correct measured isotopic ratios.

Differences in the ionization probabilities of the even mass isotopes arise from the dependence of the effective absorption cross section of the discrete transition, $\sigma_{A}^{i}$, and on the detuning of the laser center frequency from the absorption maxima of the individual isotopes, $\Delta^{i}$. We focus here on ratios of even mass isotopes because of the absence of intrinsic oddeven effects, discussed in the next section. The resonance transition of isotope $i$ is saturated if

$$
F_{\mathrm{L}} \sigma_{\mathrm{A}}^{i} \tau_{\mathrm{p}}>1
$$

where $F_{\mathrm{L}}$ and $\tau_{\mathrm{p}}$ are the laser photon flux and pulse duration, respectively. If this condition can be satisfied for two isotopes $i$ and $j$ in some laser tuning interval, $\Delta$, and for specific values of the laser bandwidth and intensity, then the measured isotope ratio, $R_{\mathrm{M}}^{i j}(\lambda)$, will be constant over this interval. When the isotopes $i$ and $j$ have known abundances, the isotope ratio measured in the region over which $R_{\mathrm{M}}^{i j}(\lambda)$ is constant may be considered to differ from the standard ratio only by purely mass-dependent fractionation. Alternatively, if the ionization maxima of the individual (even mass) isotopes are spectrally well resolved, the purely mass fractionated isotope ratios may be obtained from the ratio of the ion currents measured at the individual ionization maxima (i.e., the laser wavelength must be adjusted for each isotope).

As an example, consider the laser wavelength tuning curve for the Os $\Delta J=+1,39406.9 \mathrm{~cm}^{-1}$ resonance shown in Figure 2. ${ }^{18}$ The measured ${ }^{190} \mathrm{Os} /{ }^{188} \mathrm{Os}$ ratio is expressed as the per mil (part per thousand, denoted \%o) deviation from its standard value ${ }^{25}$ using $\delta$-notation. The ratio is constant over a wavelength interval of $0.4 \mathrm{~cm}^{-1}$, indicating the simultaneous 
saturation of the discrete transition of both isotopes. For larger detunings, isotope selective effects of up to $120 \%$ become apparent with a depletion of the heavy isotope for positive (shorter wavelength) detuning. In this experiment the UV laser bandwidth $\left[\Delta \omega_{\mathrm{L}}(\mathrm{UV})=0.4 \mathrm{~cm}^{-1}\right]$ was a factor of 8 larger than the expected optical isotope shift. ${ }^{26}$ This transition was used in a series of RIMS Os isotope experiments because under saturating conditions odd-even isotopic effects were expected to be absent. In these measurements both the even and odd mass Os isotope ratios followed a simple mass fractionation law in the region $R_{\mathrm{M}}^{i j}(\lambda)=$ constant. As pointed out above, this law is characterized by a mass independent $\alpha$ for all isotope pairs. Tuning the laser wavelength to the center of the region with ${ }^{190} \mathrm{Os} /{ }^{188} \mathrm{Os}=$ constant enabled us to measure the ${ }^{192} \mathrm{Os} /{ }^{188} \mathrm{Os}$ and ${ }^{189} \mathrm{Os} / 190 \mathrm{Os}$ ratios with a reproducibility of better than $4 \%(1 \sigma) .^{18}$

In contrast, Figure 3 presents the wavelength dependence of the individual ion intensities for ${ }^{50} \mathrm{Ti}$ and ${ }^{46} \mathrm{Ti}$ and of the ${ }^{50} \mathrm{Ti} /{ }^{46} \mathrm{Ti}$ ratio for two different $\Delta J=+1$ resonance transitions. Throughout this paper, the $\mathrm{Ti}$ isotope ratios are expressed relative to ${ }^{46} \mathrm{Ti}$ as $\delta^{i} \mathrm{Ti}=\left[\left({ }^{i} \mathrm{Ti} /{ }^{46} \mathrm{Ti}\right)_{\text {meas }} /\left({ }^{i} \mathrm{Ti} /{ }^{46} \mathrm{Ti}\right)_{\mathrm{STD}}-1\right] \times 1000$, the per mil deviation from the standard values of $\left({ }^{47} \mathrm{Ti} / 46\right.$. $\mathrm{Ti})_{\mathrm{STD}}=0.9149,\left({ }^{48} \mathrm{Ti} /{ }^{46} \mathrm{Ti}\right)_{\mathrm{STD}}=9.2125,\left({ }^{49} \mathrm{Ti} /{ }^{46} \mathrm{Ti}\right)_{\mathrm{STD}}=$ 0.6860 , and $\left({ }^{50} \mathrm{Ti} /{ }^{46} \mathrm{Ti}\right)_{\mathrm{STD}}=0.6671$ obtained by Niederer et al. ${ }^{27}$ In Figure $3 \mathrm{a}$, the position of the maximum signal at mass 46 was arbitrarily identified with zero detuning, $\Delta=0$, and the optical isotope shift between ${ }^{50} \mathrm{Ti}$ and ${ }^{46} \mathrm{Ti}$ is apparent in the distinct maxima of the individual ionization line shapes. From this measurement, we obtain $\Delta T(50-46)=0.15 \mathrm{~cm}^{-1}$, where $\Delta T$ is the value of the optical isotope shift between ${ }^{50} \mathrm{Ti}$ and ${ }^{46} \mathrm{Ti}$ for that transition. For a visible laser bandwidth of $\Delta \omega_{\mathrm{L}}=0.20 \mathrm{~cm}^{-1}$, this isotope shift produced a pronounced wavelength dependence of the measured ${ }^{50} \mathrm{Ti} /{ }^{46} \mathrm{Ti}$ ratios with variations of more than $200 \%$ for wavelength changes as small as $0.5 \Delta \omega_{\mathrm{L}}$ near the ionization maxima. It is apparent from Figure $3 a$ that for this transition there is no wavelength interval over which the ${ }^{50} \mathrm{Ti} /{ }^{46} \mathrm{Ti}$ ratio remains constant, and thus the ${ }^{50} \mathrm{Ti} /{ }^{46} \mathrm{Ti}$ ratio cannot be measured accurately at a single laser wavelength. Arbitrarily adjusting the laser wavelength to yield $\delta^{50} \mathrm{Ti}=0$ is not acceptable because this approach would produce incorrect values for the other $\mathrm{Ti}$ isotope ratios due to the offsetting effects of wavelength dependence and mass fractionation.

The resonance transition used in the experiment shown in Figure $3 \mathrm{~b}$ has a smaller optical isotope shift and a larger oscillator strength ${ }^{28}$ than the one discussed above. For this transition there is a narrow plateau extending from $\Delta \sim 0 \rightarrow$ $-0.1 \mathrm{~cm}^{-1}$ over which the ${ }^{50} \mathrm{Ti} /{ }^{46} \mathrm{Ti}$ ratio remains constant, independent of the laser wavelength. Ionization of ${ }^{46} \mathrm{Ti}$ occurs preferentially in this region, yielding $\delta^{50} \mathrm{Ti} \sim-100 \%$. The width of the plateau directly shows the accuracy of the laser wavelength setting required to obtain reproducible isotope ratio measurements. With a $f$ value of 0.4 we estimate the power broadened width of the resonance transition to lie between 1 and $0.6 \mathrm{~cm}^{-1}$, depending on the specific $M_{\mathrm{J}}$ component, close to that observed but large with respect to the measured optical isotope shift of $0.07 \mathrm{~cm}^{-1}$.

In Table I we present data for the ${ }^{50} \mathrm{Ti} /{ }^{46} \mathrm{Ti}$ and ${ }^{48} \mathrm{Ti} /{ }^{46} \mathrm{Ti}$ ratios measured in a series of experiments using different resonance transitions and laser bandwidths. For transitions in which the ${ }^{50} \mathrm{Ti} /{ }^{46} \mathrm{Ti}$ ratio exhibited a plateau as a function

(25) Creaser, R. A.; Papanastassiou, D. A.; Wasserburg, G. J. Geochim. Cosmochim. Acta 1991, 55, 397-401.

(26) Gmelin Handbook of Inorganic Chemistry, System No. 68: Platinum; Springer Verlag: New York, 1989.

(27) Niederer, F. R.; Papanastassiou, D. A.; Wasserburg, G. J. Geochim. Cosmochim. Acta 1981, 45, 1017-1031.

(28) Experimental Transition Probabilities for Spectral Lines of 70 Elements. NBS Mongr. (U.S.) 1962, No. 53.
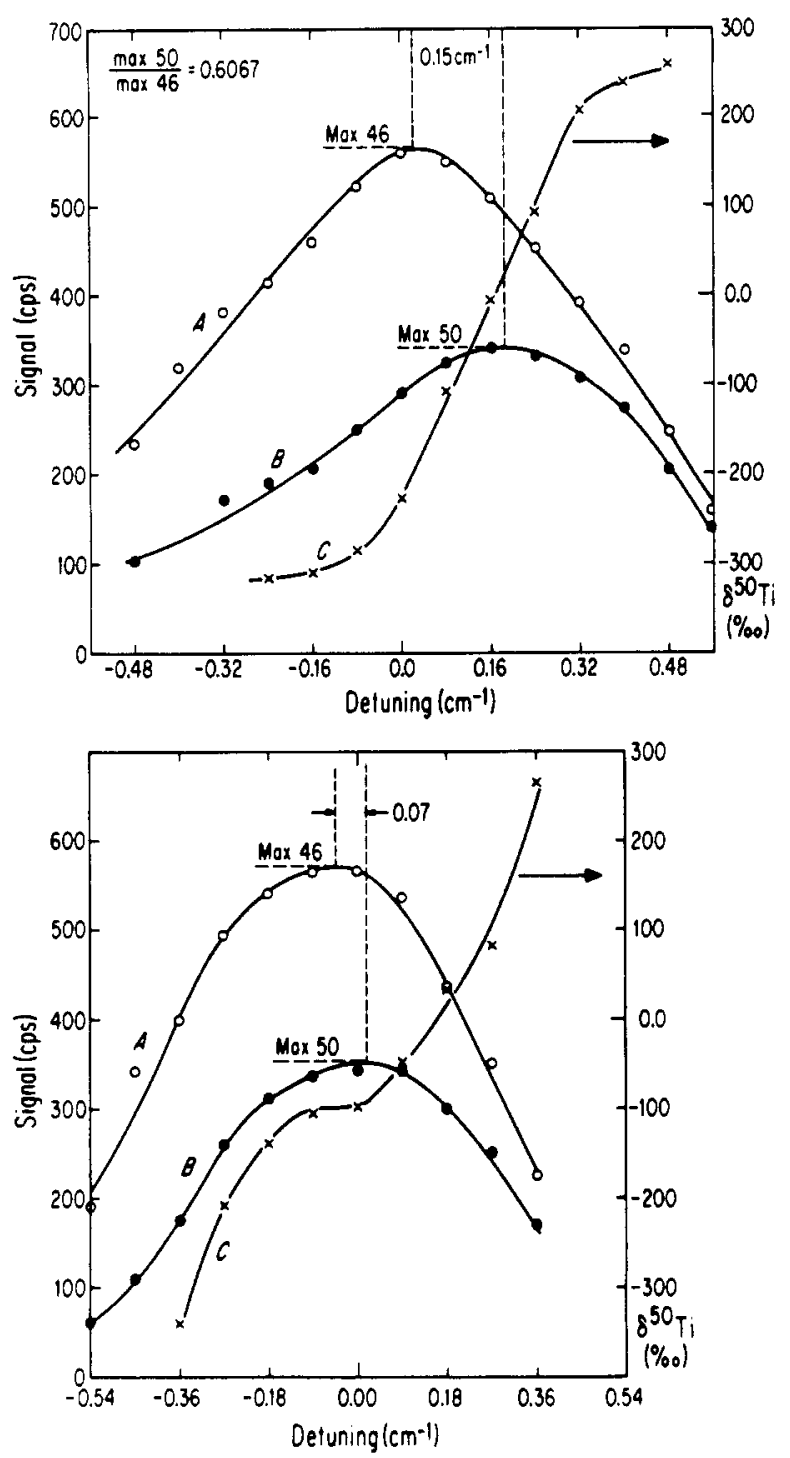

Figure 3. Wavelength dependence of the ion signal for ${ }^{46} \mathrm{Ti}$ and ${ }^{50} \mathrm{Tl}$, curves $A$ and $B$ respectively, shown on the left-hand ordinate and of the ${ }^{50} \mathrm{Ti} /{ }^{46} \mathrm{Ti}$ ratio, curve $\mathrm{C}$, on the right-hand ordinate. Typical error of the individual data points is $1 \%<1 \sigma<1.5 \%$. (a) The $\left|3 \mathrm{~d}^{2} 4 \mathrm{~s}^{2}{ }^{3} \mathrm{~F}_{2}\right\rangle$ $\rightarrow\left|3 d^{3} 4 p s^{3} D_{3}^{\circ}\right\rangle$, $39715.5 \mathrm{~cm}^{-1}$, resonance transition; oscillator strength $0.04, \Delta \omega_{L}(U V)=0.40 \mathrm{~cm}^{-1}$. (b) The $\left|3 d^{2} 4 s^{2}{ }^{3} F_{2}\right\rangle \rightarrow \mid 3 d^{2}-$ $4 s 4 p u^{3} D_{3}^{\circ}>, 38159.5 \mathrm{~cm}^{-1}$ resonance transition; oscillator strength $0.5, \Delta \omega_{\mathrm{L}}(\mathrm{UV})=0.70 \mathrm{~cm}^{-1}$

of laser wavelength, isotope ratios were calculated from ion current measurements at a laser wavelength fixed in the region $R_{\mathrm{M}}^{i j}(\lambda)=$ constant. These data are indicated as " $R(\lambda)=$ const" in Table I. For transitions where the condition $R_{\mathrm{M}}^{i j}$ $(\lambda)=$ constant could not be met, e.g., transitions shown in Figure $3 a$, isotope ratios were calculated from the ion intensities measured at the ionization maxima of the individual isotopes. These data are indicated as "peak/peak" in Table I. A comparison of the data in Table I shows that both approaches yield equivalent ${ }^{50} \mathrm{Ti} /{ }^{46} \mathrm{Ti}$ ratios, justifying the assumption that the value of the ${ }^{50} \mathrm{Ti} /{ }^{46} \mathrm{Ti}$ ratio in the plateau region (i.e., $\delta^{50} \mathrm{Ti} \sim-100 \%$ ) differs from the true value only by mass-dependent fractionation.

The mass fractionation per mass unit, $\alpha$, was obtained from the measured ${ }^{50} \mathrm{Ti} /{ }^{46} \mathrm{Ti}$ and ${ }^{48} \mathrm{Ti} /{ }^{46} \mathrm{Ti}$ ratios, assuming $f(\alpha)$ was represented by a power law. Within the measurement error, the $\alpha$ values calculated from the ${ }^{50} \mathrm{Ti} /{ }^{46} \mathrm{Ti}$ and ${ }^{48} \mathrm{Ti} /{ }^{46} \mathrm{Ti}$ ratios, shown in columns 3 and 4 of Table $I$, are in good agreement and define a mean value, $\alpha=0.025 \pm 0.002 \mathrm{amu}^{-1}$. In the remainder of the discussion, we use the value of $\alpha=$ $0.025 \mathrm{amu}^{-1}$ to correct all measured $\mathrm{T} i$ isotope ratios for mass- 
Table I. ${ }^{50} \mathrm{Ti} /{ }^{46} \mathrm{Ti}$ and ${ }^{48} \mathrm{Ti} /{ }^{46} \mathrm{Ti}$ Ratios Measured with Different Resonance Transitions

\begin{tabular}{|c|c|c|c|c|c|}
\hline resonance state $\left(\mathrm{cm}^{-1}\right)$ & ${ }^{50} \mathrm{Ti} /{ }^{46} \mathrm{Ti}( \pm 1 \sigma)$ & ${ }^{48} \mathrm{Ti} /{ }^{46} \mathrm{Ti}( \pm 1 \sigma)$ & $\alpha \times 10^{2}( \pm 1 \sigma)^{a}$ & $\alpha \times 10^{2}( \pm 1 \sigma)^{b}$ & $\operatorname{mode}^{c}$ \\
\hline $\begin{array}{c}37654.8 \\
37654.8 \\
37654.8^{h} \\
38159.5^{i} \\
39715.5 \\
39715.5 \\
39686.1 \\
\text { av }\end{array}$ & $\begin{array}{l}0.599(0.005) \\
0.604(0.006) \\
0.610(0.007) \\
0.603(0.007) \\
0.593(0.007) \\
0.607(0.009) \\
0.613(0.010) \\
0.605(0.007)\end{array}$ & $\begin{array}{l}8.791(0.14) \\
8.782(0.14) \\
8.727(0.20) \\
8.886(0.16) \\
8.552(0.20) \\
g \\
8.766(0.22) \\
8.751(0.11)\end{array}$ & $\begin{array}{l}2.7(0.2) \\
2.5(0.2) \\
2.3(0.3) \\
2.6(0.3) \\
2.9(0.3) \\
2.4(0.4) \\
2.2(0.4) \\
2.5(0.2)\end{array}$ & $\begin{array}{l}2.4(0.7) \\
2.4(0.7) \\
2.7(0.8) \\
1.8(0.8) \\
3.8(0.9) \\
g \\
2.5(1.0) \\
2.6(0.7)\end{array}$ & $\begin{array}{l}R(\lambda)=\text { const }^{d} \\
R(\lambda)=\text { const }^{d} \\
\text { peak } / \text { peak }^{e} \\
R(\lambda)=\text { const }^{d} \\
\text { peak } / \text { peak }^{d} \\
\text { peak } / \text { peak }^{d} \\
\text { peak } / \text { peak }^{e}\end{array}$ \\
\hline
\end{tabular}

a $\alpha$ calculated from measured ${ }^{50} \mathrm{Ti} /{ }^{46} \mathrm{Ti}$ ratios assuming a power law mass fractionation correction, i.e., $\alpha=\left[\left({ }^{50} \mathrm{Ti} /{ }^{46} \mathrm{Ti}\right)_{\mathrm{STD}} /\left({ }^{50} \mathrm{Ti} /{ }^{46} \mathrm{Ti}\right)_{\mathrm{meas}}\right]^{0.25}$ $-1 ;\left({ }^{50} \mathrm{Ti} /{ }^{46} \mathrm{Ti}\right)_{\mathrm{STD}}=0.6671 .{ }^{27} b \alpha$ calculated from measured ${ }^{48} \mathrm{Ti} /{ }^{46} \mathrm{Ti}$ ratios assuming a power law mass fractionation correction, $\alpha=\left[\left({ }^{48} \mathrm{Ti} /\right.\right.$ $\left.\left.{ }^{46} \mathrm{Ti}\right)_{\mathrm{STD}} /\left({ }^{48} \mathrm{Ti} /{ }^{46} \mathrm{Ti}\right)_{\text {meas }}\right]^{0.5}-1 ;\left({ }^{48} \mathrm{Ti} /{ }^{46} \mathrm{Ti}\right)_{\mathrm{STD}}=9.2125 .27 \mathrm{c} R(\lambda)=$ const indicates isotope ratios measured at a fixed wavelength for each transition. Peak/peak indicates wavelength tuned to ionization maxima for each isotope. ${ }^{d} \Delta \omega_{\mathrm{L}}$ (visible) $=0.35 \mathrm{~cm}^{-1} .{ }^{e} \Delta \omega_{\mathrm{L}}(\mathrm{visible})=0.20 \mathrm{~cm}^{-1} .{ }^{f}$ Average values calculated from the results of individual experiments. $g$ Not measured for this transition. ${ }^{h}$ Transition shown in Figure $3 \mathrm{~b} .{ }^{i}$ Transition shown in Figure $3 \mathrm{a}$.

dependent fractionation (arising from, e.g., instrument transmission, Rayleigh distillation, etc.). The ability to measure $\alpha$ reproducibly in different experiments, including changes of laser dyes and optical realignment, is due to the well-defined spatial position of the ionization volume in the ion extraction region of the ion source, as described earlier. These results indicate that resonance transitions and laser operating conditions may be found for which laser-induced isotopic selectivity is absent for the even mass $\mathrm{Ti}$ isotopes. Further, in the wavelength regions over which the ${ }^{50} \mathrm{Ti} /{ }^{46} \mathrm{Ti}$ ratio is independent of wavelength, the fractionation law must also be valid for the odd mass $\mathrm{Ti}$ isotopes. Any deviations in even-odd $\mathrm{Ti}$ isotope ratios, measured in these wavelength regions, remaining after correction for instrumental mass fractionation must, therefore be due to intrinsic, laser-induced even-odd effects in the resonance ionization process.

As Figures 2 and 3 demonstrate, the wavelength effects in $T i$ are significantly different in sign and magnitude from the effects observed in Os. The absorption maxima of the heavier Os isotopes are shifted to longer wavelengths. This "normal" shift reflects the increase of the average squared nuclear charge radius in $\mathrm{Os}$ upon addition of neutrons. ${ }^{29}$ In $\mathrm{Ti}$, the wavelength shift is reversed. The enrichment of ${ }^{50}$ Ti measured at shorter wavelengths (Figure 3 ) reflects a decrease in the nuclear charge radius approaching a closed neutron shell with magic neutron number $28 .{ }^{30}$

The field shift contribution to the IS is proportional to the change of the electron charge density at the nucleus in the relevant electronic states. Thus, optical isotope shifts are most pronounced in transitions involving s electrons. Ti has the ground-state electron configuration $3 \mathrm{~d}^{2} 4 \mathrm{~s}^{2}$, the lowest term is $\left|{ }^{3} \mathbf{F}\right\rangle$. The resonant excited state in the experiment shown in Figure $3 a$ has the electron configuration and level $\mid 3 d^{3} 4 p$ $\mathrm{s}^{3} \mathrm{D}_{3}^{\mathrm{o}}>$, while the resonant state used for the experiment shown in Figure $3 \mathrm{~b}$ has the electron configuration and level $\left|3 \mathrm{~d}^{2} 4 \mathrm{~s} 4 \mathrm{p} \mathrm{u}^{3} \mathrm{D}_{3}^{o}\right\rangle$. Optical isotope shifts were much more pronounced in the first case because the transition involves the removal of two s electrons. Table II shows a list of the optical isotope shifts $\Delta T(50-46)$ measured for several resonance transitions. Note the difference in the IS between the $3 d^{3} 4 p$ and the $3 d^{2} 4 s 4 p$ excited-state configurations.

The wavelength dependence of $\mathrm{Os}$ isotope ratios is less pronounced compared to that observed in Ti because (a) the change in the average nuclear charge radius with neutron number is larger for $\mathrm{Ti}^{29,30}$ and (b) the resonance transitions we studied in Os involve 6s electrons, which possess a much lower electron density at the nucleus compared to those studied in $\mathrm{Ti}$. We expect these effects to be less significant

(29) Wohlfahrt, H. D.; Shera, E. B.; Hoehn, M. V.; Yamazaki, Y.; Steffen, R. M. Phys. Rev. C 1981, 23, 533-548.

(30) Hoehn, M. V.; Shera, E. B. Phys. Rev. C 1979, 20, 1934-1941.
Table II. Optical Isotope Shifts between ${ }^{50} \mathrm{Ti}$ and ${ }^{46} \mathrm{Ti}$ for Different Resonance Transitions

\begin{tabular}{ccc}
\hline $\begin{array}{c}\text { resonance } \\
\text { frequency }\left(\mathrm{cm}^{-1}\right)\end{array}$ & $\begin{array}{c}\text { excited-state } \\
\text { configuration and level }\end{array}$ & $\begin{array}{c}\text { isotope shift }\left(\mathrm{cm}^{-1}\right) \\
\Delta T(50-46)( \pm 1 \sigma)\end{array}$ \\
\hline 37654.8 & $\left|3 \mathrm{~d}^{3} 4 \mathrm{p} \mathrm{u}^{3} \mathrm{~F}_{2}^{0}\right\rangle$ & $0.21(0.03)$ \\
$37852.5^{a}$ & $\left|3 \mathrm{~d}^{3} 4 \mathrm{p} \mathrm{u}^{3} \mathrm{~F}_{4}^{0}\right\rangle$ & $0.14(0.02)$ \\
38159.7 & $\left|3 \mathrm{~d}^{2} 4 \mathrm{~s}^{0} \mathrm{p} \mathrm{u}^{3} \mathrm{D}_{3}^{0}\right\rangle$ & $0.07(0.02)$ \\
39686.1 & $\left|3 \mathrm{~d}^{3} 4 \mathrm{p} \mathrm{s}^{3} \mathrm{D}_{2}^{0}\right\rangle$ & $0.18(0.02)$ \\
39715.5 & $\left|3 \mathrm{~d}^{3} 4 \mathrm{p} \mathrm{s}^{3} \mathrm{D}_{3}^{0}\right\rangle$ & $0.15(0.02)$
\end{tabular}

a Transition from the $\left|a^{3} F_{3}\right\rangle, 170 \mathrm{~cm}^{-1}$ excitation, ground-state spinorbit level. All other lines involve the $\left|a^{3} \mathrm{~F}_{2}\right\rangle$ true ground-state spinorbit level.

in the Ti RIMS measurements by Spiegel et al..$^{10,11}$ due to the much larger Doppler width induced by secondary neutral atom sputtering in their experiments and their selection of an excited $3 d^{2} 4 s 4$ p electron configuration in the first resonance step.

\section{ODD-EVEN ISOTOPE EFFECTS}

Several physical effects can produce different ionization efficiencies of atoms with and without nuclear spin in a resonance ionization process. In this discussion, we assume $\Delta \omega_{\mathrm{L}}>\Delta \omega_{\mathrm{HFS}}$, where $\Delta \omega_{\mathrm{HFS}}$ is the spectral width of the hyperfine array. We distinguish dynamic effects, including quantum mechanical coherence, ${ }^{14-17}$ from selection rule and polarization effects. ${ }^{9,10,18,19}$ Some of these effects can be visualized by the linkage diagrams shown in Figure 4a,b which represent $J=0 \leftrightarrow J^{\prime}=1$ transitions for an atom with nuclear spin $I=0$ and $I=1 / 2$, respectively. The angular momentum states of the atom with nuclear spin are represented as usual by $\left|J I F M_{\mathrm{F}}\right\rangle$, with $F=J+I$. Consider a resonance ionization process with a pulse duration $\tau_{p}$, an inverse ionization rate $1 / R_{\mathrm{I}} \gg \Delta \omega_{\mathrm{HFS}}$, and a laser bandwidth $\Delta \omega_{\mathrm{L}}>\Delta \omega_{\mathrm{HFS}}$. Under these conditions, which are met in many resonance ionization experiments, equilibration of ground- and excited-state populations occurs early in the laser pulse for all allowed transitions. From the $\Delta J=+1$ linkage diagram in Figure $4 a$, it is easy to see that, for linearly polarized light and $I=0$, half of the total atomic population will be in the excited state, from which ionization proceeds at a much slower rate. ${ }^{31}$ Due to the increase of available angular momentum states, the corresponding excited-state population for $I=1 / 2$ increases to two-thirds. If the ionization step is not saturated, i.e., $\tau_{\mathrm{p}} R_{\mathrm{I}}$ $<1$, the number of ions generated from atoms with and without nuclear spin can be considerably different with an enhancement of the odd mass isotopes. Under saturating ionization conditions, this difference tends to zero. ${ }^{14,17,18}$ In

(31) Hurst, G. S.; Payne, M. G.; Kramer, S. D.; Young, J. P. Rev. Mod. Phys. 1979, 51, 767-819. 


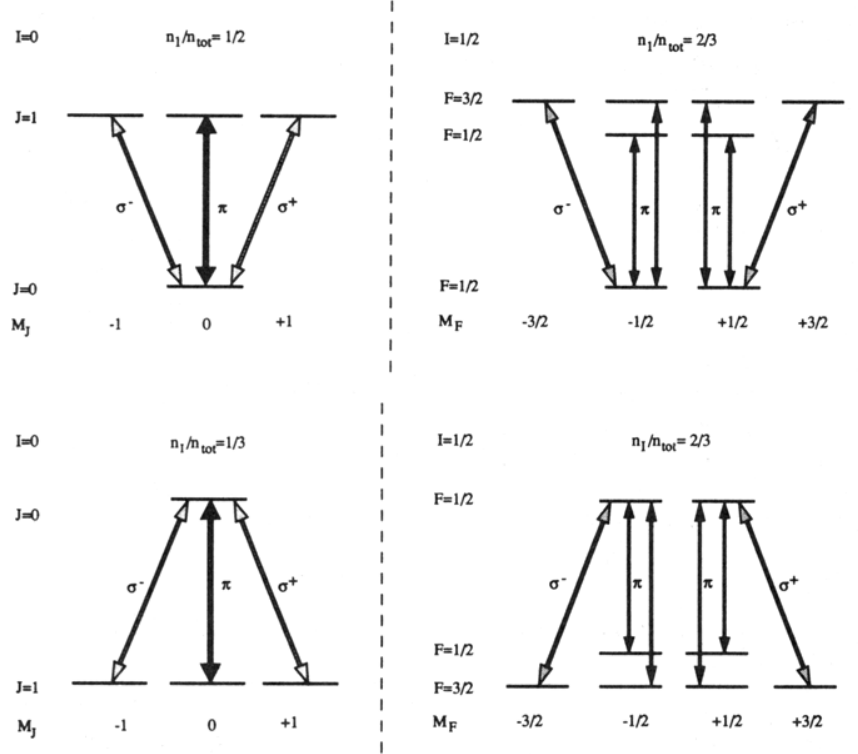

Flgure 4. (a) Linkage diagram for a $J=0 \rightarrow J^{\prime}=1$ dipole transition for atoms with $(I=1 / 2)$ and without $(I=0)$ nuclear spin. $n_{1} / n_{\text {tot }}$ represents the fractional, steady-state saturated excited-state population for excitation with linearly polarized light. $n_{\text {tot }}$ is the number density of a specific isotope before onset of interaction. The laser bandwidth is larger than the splitting between the $F=1 / 2$ and $F=3 / 2$ HFS states. (b) Linkage diagram for a $J=1 \rightarrow J^{\prime}=0$ dipole transition for atoms with $(I=1 / 2)$ and without $(I=0)$ nuclear spin. $n_{1} / n_{\text {tot }}$ represents the fractional saturated ionization yield for excitation with linearly polarized light. $m_{\text {tot }}$ is the number density of a specific isotope before onset of interaction.

Table III. ${ }^{47} \mathbf{T i} /{ }^{46} \mathrm{Ti}$ and ${ }^{49} \mathrm{Ti} /{ }^{46} \mathrm{Ti}$ Ratios for Three Different $\Delta J=+1$ Transitions

\begin{tabular}{cccl}
\hline $\begin{array}{c}\text { resonance } \\
\text { state, } \nu\left(\mathrm{cm}^{-1}\right)\end{array}$ & $\delta^{47} \mathrm{Ti}( \pm 1 \sigma)^{a}$ & $\delta^{49} \mathrm{Ti}( \pm 1 \sigma)^{a}$ & \multicolumn{1}{c}{ mode $^{b}$} \\
\hline${ }^{3} \mathrm{~F}_{3}, 38544.4$ & $-14(12)$ & $3(11)$ & saturation \\
${ }^{3} \mathrm{~F}_{4}, 37852.5$ & $35(11)$ & $5(11)$ & saturation \\
${ }^{3} \mathrm{D}_{3}, 38159.5$ & $60(15)$ & $144(23)$ & nonsaturation
\end{tabular}

${ }^{a} \delta^{i} \mathrm{Ti}(\%)=\left[\left({ }^{i} \mathrm{Ti} /{ }^{46} \mathrm{Ti}\right)_{\text {meas }} /\left({ }^{i} \mathrm{Ti} /{ }^{46} \mathrm{Ti}\right)_{\mathrm{STD}}-1\right] \times 1000 . \quad \mathrm{The}\left({ }^{47} \mathrm{Ti} /\right.$ $\left.{ }^{46} \mathrm{Ti}\right)_{\mathrm{STD}}$ and $\left({ }^{49} \mathrm{Ti} /{ }^{46} \mathrm{Ti}\right)_{\mathrm{STD}}$ ratios are 0.9149 and 0.6860 , respectively. ${ }^{27}$ ${ }^{b}$ Indicates if the ionization step was saturated, i.e., $\tau_{\mathrm{p}} R_{\mathrm{I}}>1$.

addition, the photoionization cross sections of the individual $\mid J I F M_{\mathrm{F}}>$ and $\mid L S J M_{\mathrm{J}}>$ components of the excited state will be somewhat different and require saturation of the ionization process in order to avoid laser-induced odd-even isotopic bias.

For the measurement of odd-even isotope effects, the laser wavelength was tuned such that the ${ }^{48} \mathrm{Ti} /{ }^{46} \mathrm{Ti}$ ratio had the purely mass fractionated value given in Table $\mathrm{I}$ in order to assure that this ratio was free of laser wavelength tuning effects. Table III shows the results of measurements of the ${ }^{47} \mathrm{Ti} /{ }^{46} \mathrm{Ti}$ and ${ }^{49} \mathrm{Ti} /{ }^{46} \mathrm{Ti}$ ratios (expressed as $\delta^{47} \mathrm{Ti}$ and $\delta^{49} \mathrm{Ti}$, respectively ${ }^{27}$ ), measured on three $\Delta J=+1$ transitions. The ratios have been corrected for instrumental mass fractionation as described above, and we verified that the hyperfine structure of the odd mass isotopes could not be resolved within the laser bandwidth in all experiments. With the exception of the $\delta^{47} \mathrm{Ti}$ value of $35 \%$ for the second transition listed, the data collected under saturating ionization conditions show little evidence of sizable odd-even effects. In contrast, the data obtained under nonsaturating conditions (last row of Table III) exhibit large deviations from normal values for both the ${ }^{47} \mathrm{Ti} /{ }^{46} \mathrm{Ti}$ and ${ }^{49} \mathrm{Ti} /{ }^{46} \mathrm{Ti}$ ratios, $\delta^{47} \mathrm{Ti}=60 \pm 15 \%$ and $\delta^{49} \mathrm{Ti}=144 \pm 23 \%$. These laser-induced effects far exceed the analytical error and are similar to isotope shifts found for other systems under nonsaturating conditions. ${ }^{9}$
We note that the difference between $\delta^{47} \mathrm{Ti}$ and $\delta^{49} \mathrm{Ti}$ shown in the last row of Table III may be due to the larger number of excited-state components for ${ }^{49} \mathrm{Ti}(I=5 / 2)$, i.e., 42 , compared to 28 for ${ }^{47} \mathrm{Ti}(I=3 / 2)$. In a steady state, more ${ }^{49} \mathrm{Ti}$ than ${ }^{47} \mathrm{Ti}$ atoms are in the excited state. This is analogous to the case shown schematically in Figure 4a. The Ti data demonstrate that odd-even laser-induced isotopic selectivity can be reduced below the $2 \%$ level in the case of a $1+1$ ionization scheme for selected $\Delta J=+1$ transitions and linearly polarized light. The same principles should apply to three-photon, two-color resonant ionization schemes, although the details of the dynamical equations are much more complex. ${ }^{15}$

Payne et al. ${ }^{17}$ recently predicted that, for realistic spatial intensity distributions of the ionizing laser radiation, some odd mass isotope enrichment due to photoion production in regions where the ionization is not saturated will always occur. This enrichment was calculated to be approximately $3 \%$ for $\mathrm{Sn}$, depending on the detailed laser beam parameters. It may be possible to avoid this residual odd-even effect by using specially designed ion extraction optics, laser detuning, ${ }^{17,32}$ or fast photoionization. ${ }^{14}$ It also appears that, whenever the radius of the ionization laser beam is larger than that of the excitation laser, the ionization can be saturated in all regions where atoms are in an excited state. In this case, precise beam tailoring may provide another technique to avoid isotopic selectivity related to edge effects.

In the case of fast ionization, i.e. $R_{\mathrm{I}} \gg \Delta \omega_{\mathrm{HFS}}$ and/or if $\tau_{\mathrm{p}}$ $<\Delta \omega_{\mathrm{HFS}^{-1}}$, the ionization process may be visualized as originating from a coherent superposition of the excited-state hyperfine components. ${ }^{33-35}$ This situation is closely related to quantum beat spectroscopy. For a time smaller than the hyperfine coupling period, the whole excited-state population resides in one specific $F$ state with half the atomic population in the excited state from which it will be ionized. Thus, with regard to population balance, excitation and ionization proceed as if there were no hyperfine structure. ${ }^{14,17,36}$ Isotopic selectivity may, therefore, be avoided by choosing interaction times shorter than the hyperfine coupling time, $1 / \Delta \omega_{\mathrm{HFS}}$. However, in many practical applications it is difficult to saturate the ionization in time intervals shorter than $10^{-9} \mathrm{~s}$. The resulting high peak power and increased laser bandwidth, even for transform-limited ultrafast pulses, may also reduce significantly the selective ionization properties of RIMS in complex natural samples where a variety of elements are present.

Frequency domain coherent techniques may provide another route to the elimination of isotopic selectivity in RIMS, but require very complex lasers. Alternatively, following the linkage diagram presented in Figure $4 \mathrm{a}$, we suggest that depolarization of the laser radiation, producing equal excitedstate populations for the even and odd mass isotopes, could offer a simple means of reducing even-odd effects for $\Delta J=$ +1 transitions. Selection rule and polarization effects for $\Delta J$ $=-1$ transitions may be visualized using the linkage diagram shown in Figure $4 \mathrm{~b}$, which presents the specific $J=1 \rightarrow J^{\prime}$ $=0$ case. For linearly polarized light, $67 \%$ of the groundstate population of the even mass isotope, $I=0$, does not interact with the laser field, while for the odd mass isotope

(32) Brandon, W.; Alman, S. L.; Payne, M. G.; Garrett, W. R.; Chen C. H. Presented at the VI International Symposium on Resonance Ionization Spectroscopy and its Applications, Sante Fe, NM, May 24-29, 1992.

(33) Georges, A. T.; Lambropoulos, P. Phys. Rev. A 1978, 18, 10721078 .

(34) Leuchs, G.; Smith, S. J.; Khawaja, E.; Walther, H. Opt. Commun. 1979, 31, 313-316.

(35) Ducas, T. W. Littman, M. G.;Zimmerman, M. L. Phys, Rev. Lett. $1975,35,1742-1754$.

(36) Shore, B. W.; Johnson, M. A. Phys. Rev. A 1981, 23, 1608-1610. 
with $I=1 / 2$, the corresponding number is $33 \%$, leading to a $100 \%$ enrichment of the odd mass isotope during ionization. In Figure $4 a, b$ the different HFS components of the resonance transition are indicated by individual lines, but we emphasize that the laser spectrum overlaps these transitions continuously. If the laser field has a multimode structure, several HFS ground-state components can, in principle, interact with a common excited state via different laser modes. This situation has recently been studied theoretically and experimentally by Whitten and Ramsey ${ }^{16}$ using $\mathrm{CW}$ excitation of $\mathrm{Na}$ atoms. Coherent population trapping reduces the excitation of the isotope with nuclear spin when a certain velocity group is simultaneously resonant with two or more HFS ground-state components. The size of the coherent dip in the resonance excitation spectrum depends critically on the laser bandwidth, and this effect will be absent with large bandwidth pulsed laser excitation.

Excitation with depolarized light can avoid this type of odd mass isotope enrichment because in all cases one-fourth of the atoms of each isotope will be in the excited state from which they are ionized. In many practical situations the polarization state of the laser is not well characterized, and the measured isotope ratios will depend on the detailed spatial intensity distribution of the different polarization components and the saturation behavior of the transitions associated with these components. ${ }^{18}$

In order to address these concerns, we have examined the $\delta^{47} \mathrm{Ti}$ and $\delta^{49} \mathrm{Ti}$ values for the $\Delta J=0,\left|3 \mathrm{~d}^{2} 4 \mathrm{~s}^{2} \mathrm{a}^{3} \mathrm{~F}_{2}\right\rangle \rightarrow \mid 3 \mathrm{~d}^{3} 4 \mathrm{p}$ $\mathrm{u}^{3} \mathrm{~F}_{2}^{\circ}>37654.8 \mathrm{~cm}^{-1}$ transition with two different degrees of depolarization of the laser beam. The degree of depolarization, $P_{\text {ell }}$, is defined as the ratio of the maximum to the minimum intensity in the laser radiation, measured as a function of the rotation angle of a linear polarizer. The depolarization was generated by passing the laser beam through a stressed quartz window, thus inducing a random amount of birefringence across the laser beam waist. The polarization measurements were performed for saturating laser intensities with regard to excitation and ionization, but the Stokes parameters of the laser fields could not be measured. A laser wavelength setting which reproduced the purely mass fractionated ${ }^{48} \mathrm{Ti} /{ }^{46} \mathrm{Ti}$ ratio was used. These laser tuning conditions yielded the maximum signal for ${ }^{47} \mathrm{Ti}$, assuring that the ${ }^{47} \mathrm{Ti} /{ }^{46} \mathrm{Ti}$ ratio is free of laser wavelength tuning bias.

For a high degree of linear polarization, the data show a large odd mass isotope enrichment, which is greatly reduced when the laser radiation becomes more depolarized. Specifically, with $P_{\text {ell }}=220$ and $\Delta \omega_{\mathrm{L}}(\mathrm{UV})=0.7 \mathrm{~cm}^{-1}$, we found $\delta^{47} \mathrm{Ti}$ $=137 \pm 10 \%$ and $\delta^{49} \mathrm{Ti}=135 \pm 10 \%$. Two separate experiments with $P_{\text {ell }}=23$ yielded $\delta^{47} \mathrm{Ti}=2.7 \pm 10 \%$ and 16 $\pm 15 \%$. These latter measurements were carried out with a smaller $\Delta \omega_{L}(\mathrm{UV})=0.4 \mathrm{~cm}^{-1}$ and with the laser wavelength tuned to yield the maximum signal at mass 47 . The $\delta^{49} \mathrm{Ti}$ values, therefore, show additional wavelength-induced isotopic bias and cannot be directly compared with $\delta^{47} \mathrm{Ti}$. We wish to point out, however, that the small shift $(\Delta=0.09$ $\mathrm{cm}^{-1}$ ) in laser wavelength between the two experiments is considerably less than the observed IS of $0.21 \mathrm{~cm}^{-1}$.

While a beam with $P_{\text {ell }}=23$ is clearly different than a truly depolarized beam, if saturation is achieved in both polarization states, the results are equivalent to the ideal case of $P_{\text {ell }}=1$. Indeed, the reduction of the odd mass isotope enrichment of $P_{\text {ell }}=23$ can be qualitatively explained by the saturation of the $M_{\mathrm{J}}=0 \rightarrow M_{\mathrm{J}^{\prime}}= \pm 1$ transition due to the presence of approximately $5 \%$ circularly polarized light in the laser field, where $J$ and $J^{\prime}$ represent the total angular momentum of the ground and excited state. Consider a Rabi frequency $\Omega_{\mathrm{L}}$ connecting the $M_{\mathrm{J}}=1$ and $M_{\mathrm{J}^{\prime}}=1$ states with linearly polarized light. We obtain $\Omega_{\mathrm{L}}=1.01 \times 10^{7}\left(J_{\mathrm{L}}\right)^{1 / 2} \mathrm{rad} / \mathrm{s}$, where $J_{\mathrm{L}}$ is the linearly polarized laser intensity (in $W / \mathrm{cm}^{2}$ ). For the corresponding Rabi frequency $\Omega_{\mathrm{C}}$ connecting the $M_{\mathrm{J}}=0$ and $M_{J^{\prime}}= \pm 1$ states with circularly polarized light, we calculate $\Omega_{\mathrm{C}}=1.43 \times 10^{7}\left(J_{\mathrm{C}}\right)^{1 / 2} \mathrm{rad} / \mathrm{s}$. An oscillator strength of $5.8 \times$ $10^{-3}$ has been used. ${ }^{28}$ The transition rate can be expressed as $R=\Omega^{2} / 2 \pi \Gamma_{\mathrm{L}}$, where $R$ and the laser bandwidth $\Gamma_{\mathrm{L}}$ have units of $\mathrm{s}^{-1}$ and $\mathrm{rad} / \mathrm{s}$, respectively. ${ }^{31}$ With $\Delta \omega_{\mathrm{L}}(\mathrm{UV})=0.4$ $\mathrm{cm}^{-1}$, we find $R_{\mathrm{L}}=1.35 \times 10^{3} J_{\mathrm{L}} \mathrm{s}^{-1}$ and $R_{\mathrm{C}}=2.71 \times 10^{3} J_{\mathrm{C}}$ $\mathrm{s}^{-1}$. The discrete transitions are saturated if $R_{\mathrm{L}, \mathrm{C}} \tau_{\mathrm{P}}>1$. Under our typical conditions of a UV intensity of $2.5 \times 10^{6} \mathrm{~W} / \mathrm{cm}^{2}$, $P_{\text {ell }}=23$, and $R_{\mathrm{C}} \tau_{\mathrm{P}} \approx 6$, the transition driven by the minor circularly polarized UV component is saturated. This is no longer the case when $P_{\text {ell }}$ is as large as 220 .

However, even for $P_{\text {ell }}=220$ there will be some excitation of the $\Delta M_{\mathrm{J}}= \pm 1$ transitions, reducing the odd mass isotope enrichment compared to that predicted for purely linearly polarized light. The size of the effect depends on the laser intensity and the oscillator strength of the transition. For a truly quantitative evaluation, the spatial intensity distribution of the laser beam must be taken into account. ${ }^{18}$ More complete studies, in which the polarization state of the laser may be continuously and precisely varied, will be necessary to characterize fully the potential of depolarized light sources in RIMS, but our results suggest that they may offer a convenient means of reducing laser-induced isotopic bias.

In a $\Delta J=0$ transition with integer electron angular momentum and with linearly polarized light, odd-even isotopic selectivity is caused by the forbidden $M_{\mathrm{J}}=0 \rightarrow M_{\mathrm{J}^{\prime}}$ $=0$ transition. ${ }^{13}$ This selection rule is not applicable for the half-integer total angular momentum of the odd mass isotopes. The expected odd mass isotope enhancement for this transition, calculated from the corresponding linkage diagram and $P_{\text {ell }}=\infty$, is $\delta^{47} \mathrm{Ti}=250 \%$. This value should be independent of nuclear spin (i.e., $\delta^{49} \mathrm{Ti}$ should equal $\delta^{47} \mathrm{Ti}$ ). The observed isotope shift is smaller than expected, due to the partial saturation of the $\Delta M_{\mathrm{J}}=0$ transition by a small degree of depolarization (or elliptical polarization) in the laser radiation. The reduction of the odd-even isotopic selectivity for a $\Delta J=0$ transition with increasing degree of polarization is consistent with our Os RIMS isotope data. ${ }^{18}$

The absence of laser-induced isotope selectivity for the ${ }^{47} \mathrm{Ti} /{ }^{46} \mathrm{Ti}$ and ${ }^{49} \mathrm{Ti} /{ }^{46} \mathrm{Ti}$ ratios under saturating ionization conditions found here is distinctly different from the large enhancements of ${ }^{47} \mathrm{Ti}$ and ${ }^{49} \mathrm{Ti}$ reported by Spiegel et al.,10,11 who utilized a three-photon, two-color resonance ionization scheme with an autoionizing state and a series of $\Delta J=0$ discrete transitions. While the measured ${ }^{46} \mathrm{Ti} /{ }^{48} \mathrm{Ti}$ and ${ }^{50} \mathrm{Ti} /{ }^{48} \mathrm{Ti}$ ratios are close to the expected values and exhibit no consistent mass-dependent fractionation, the abundances of ${ }^{47} \mathrm{Ti}$ and ${ }^{49} \mathrm{Ti}$ are strongly enhanced (e.g., $\delta^{47} \mathrm{Ti}=325 \%$ and $\delta^{49} \mathrm{Ti}=450 \%$ for a $\mathrm{Ti}$ metal target). These enrichments are much larger than that expected from a series of $\Delta J=0$ transitions for linearly polarized light, i.e., $\delta^{47} \mathrm{Ti}=\delta^{49} \mathrm{Ti}=$ $250 \%$. Dynamic effects, which have been treated theoretically by Lyras et al. ${ }^{15}$ for multistep resonance ionization involving $\Delta J=+1$ transitions, should be greatly reduced under saturating ionization conditions, as was the case in the study of Spiegel et al. Thus, it appears possible that the autoionizing step may itself introduce additional odd-even isotopic selectivity. Autoionizing states are characterized by specific angular momentum quantum numbers, and additional isotopic selectivity could be introduced if the autoionizing step involves a $\Delta J=-1$ transition. This could also qualitatively explain the observed difference in $\delta^{47} \mathrm{Ti}$ and $\delta^{49} \mathrm{Ti}$, with $\delta^{49} \mathrm{Ti}$ $>\delta^{47} \mathrm{Ti}$. As autoionizing resonances are often used in RIMS experiments for enhancement of the ionization efficien- 
$\mathrm{cy}_{,}^{3,4,37,38}$ the possible influence of autoionizing transitions on odd-even isotopic selectivity clearly requires further experimental investigation. Excitation of high-lying Rydberg levels followed by pulsed-field ionization may offer a more robust means of enhancing the ionization efficiency, while also providing another degree of elemental selectivity.

\section{CONCLUSIONS}

We have presented an analysis of isotopic selectivity in the $1+1$ resonance ionization of $\mathrm{Ti}$. The measured $\mathrm{Ti}$ isotope ratios depend on four important parameters of the RIMS process: (1) the precise laser wavelength and resetability; (2) the type of transition with regard to angular momentum change; (3) the degree of saturation of the transition; and (4) the polarization state of the laser. Failure to take into account the influence of these parameters on RIMS isotope ratio measurements may cause large laser-induced isotopic shifts, which may in turn hinder the application of RIMS to high precision isotope ratio mass spectrometry. The pronounced wavelength dependence of the $\mathrm{Ti}$ isotope ratios is caused by large optical isotope shifts, reflecting the decrease in nuclear volume approaching the magic neutron number 28 in ${ }^{50} \mathrm{Ti}$. Wavelength-dependent measurements of even mass Ti isotope ratios provide information about the isotope shifts of the resonance transition and identify certain transitions for which the effect is minimal. For selected transitions and with a suitable choice of laser operating parameters, the even mass $\mathrm{Ti}$ isotopes follow a simple mass-dependent fractionation pattern; measured ${ }^{48} \mathrm{Ti} /{ }^{46} \mathrm{Ti}$ and ${ }^{50} \mathrm{Ti} /{ }^{46} \mathrm{Ti}$ ratios yield a constant mass fraction factor, $\alpha=0.025 \pm 0.002 \mathrm{amu}^{-1}$. We find that ratios of the even mass $T i$ isotopes can be measured simultaneously when the laser bandwidth or power broadened width of the transition exceeds the optical isotope shift by a factor of 8-10. Alternatively, the laser wavelength can be tuned to the ionization maxima of the individual isotopes. ${ }^{12}$ This latter procedure does complicate the experimental procedure, however.

With regard to laser-induced isotopic selectivity for the odd mass isotopes, a $\Delta J=+1$ resonance transition and for saturating laser intensity in the ionization step, our data indicate (with the exception of one value for $\delta^{47} \mathrm{Ti}$ ) the absence of significant odd-even effects.

(37) Rimke, H.; Hermann, G.; Mang, M.; Mühleck, C.; Riegel, J.; Sattelberger, P.; Trautmann, N.;Ames, F.; Becker, A.; Kluge, H.-J.; Monz L.;Otten, E. W.; Rehlau, D.; Ruster, W. Resonance Ionization Spectroscopy 1988. Inst. Phys. Conf. Ser. 1988, No. 94, 351-356.

(38) Krönert, U.; Becker, S.; Hilberath, T.; Kluge, H.-J.; Schultz, C. Appl. Phys. A 1987, 44, 339-345.
Coherent excitation with ultrafast pulses or specially tailored nanosecond pulse sequences may yield high ionization efficiency with little laser-induced isotopic selectivity, but an examination of these techniques will require considerably more sophisticated laser technology than that utilized here. Polarization scrambling offers another possibility for the measurement of unbiased isotope ratios with the advantage of simple instrumentation and a higher versatility in the choice of a resonance ionization scheme for a particular element. For example, with a $\Delta J=0$ transition and a depolarized laser beam, the odd mass isotope enrichment was reduced below the $2 \%$ level. This is an important step in the development of a multielement capability in isotopic analysis using RIMS.

From our observations, we conclude that it is necessary to study the effects described above spectroscopically for each ionization scheme being considered for analytical purposes, avoiding those transitions which either obviously or subtly cause large isotopic selectivity. In particular, the effect of autoionizing resonances on measured odd-even isotope ratios requires careful consideration.

The results of these Ti RIMS experiments are in good agreement with the results of our previous Os RIMS experiment. The data for both elements demonstrate that by carefully choosing resonance transitions and laser operating parameters, isotope ratios involving both even and odd mass isotopes, free from laser-induced isotope selectivity at the level of counting statistics, can be measured using RIMS. These two studies are among the few RIMS experiments to demonstrate this important result, and we believe that such techniques should enable RIMS to determine unbiased isotope ratios in natural samples with a precision and accuracy of better than $1 \%$.

\section{ACKNOWLEDGMENT}

We thank D. A. Papanastassiou and P. G. Green for assistance in these experiments and M. E. Johnson for manuscript preparation. This work was supported by DOE Grant DE FG03-88ER-13851 (to G.J.W.) and NASA Grant NAGW-1944 (to G.A.B.). The laser system was obtained through support from the David and Lucille Packard and Alfred P.Sloan Foundations (G.A.B.). Division Contribution 5078(749).

RECEIVED for review July 6, 1992. Accepted February 4, 1993. 